\title{
Thinning and drilling laser-assisted hatching in thawed embryo transfer: A randomized controlled trial
}

\author{
Minh Tam Le 1,2, Thi Tam An Nguyen', Thi Thai Thanh Nguyen', Van Trung Nguyen', Dinh Duong Le', Vu Quoc Huy Nguyen², \\ Ngoc Thanh Cao ${ }^{1,2}$, Alar Aints ${ }^{4}$, Andres Salumets ${ }^{4,5,6,7}$ \\ ${ }^{1}$ Center for Reproductive Endocrinology and Infertility (HUECREI), Hue University of Medicine and Pharmacy, Hue; ${ }^{2}$ Department of Obstetrics and \\ Gynecology, Hue University of Medicine and Pharmacy, Hue; ${ }^{3}$ Department of Public Health, Hue University of Medicine and Pharmacy, Hue, Vietnam; \\ ${ }^{4}$ Institute of Clinical Medicine, Department of Obstetrics and Gynecology, University of Tartu, Tartu; ${ }^{5}$ Competence Centre on Health Technologies, Tartu; \\ ${ }^{6}$ Institute of Biomedicine and Translational Medicine, University of Tartu, Tartu, Estonia; ${ }^{7}$ Department of Obstetrics and Gynaecology, University of \\ Helsinki and Helsinki University Hospital, Helsinki, Finland
}

Objective: In frozen and thawed embryos, the zona pellucida (ZP) can be damaged due to hardening. Laser-assisted hatching (LAH) of embryos can increase the pregnancy rate. This study compared thinning and drilling of the ZP before frozen embryo transfer (FET).

Methods: Patients were randomly allocated into two groups for LAH using thinning or drilling on day 2 after thawing. Twenty-five percent of the ZP circumference and $50 \%$ of the ZP thickness was removed in the thinning group, and a hole $40 \mu \mathrm{m}$ in diameter was made in the drilling group.

Results: A total of 171 in vitro fertilization/intracytoplasmic sperm injection FET cycles, including 85 cycles with drilling LAH and 86 cycles with thinning $\mathrm{LAH}$, were carried out. The thinning group had a similar $\beta$-human chorionic gonadotropin-positive rate $(38.4 \%$ vs. $29.4 \%)$, implantation rate $(16.5 \%$ vs. $14.4 \%)$, clinical pregnancy rate $(36.0 \%$ vs. $25.9 \%)$, miscarriage rate $(5.8 \%$ vs. $2.4 \%)$, ongoing pregnancy rate $(30.2 \%$ vs. $23.5 \%)$, and multiple pregnancy rate (7.0\% vs. $10.6 \%)$ to the drilling LAH group. There were no significant differences in pregnancy outcomes between subgroups defined based on age (older or younger than 35 years) or ZP thickness (greater or less than $17 \mu \mathrm{m}$ ) according to the LAH method.

Conclusion: The present study demonstrated that partial ZP thinning or drilling resulted in similar outcomes in implantation and pregnancy rates using thawed embryos, irrespective of women's age or ZP thickness.

Keywords: Assisted reproductive techniques; Laser assisted hatching; Zona pellucida

\section{Introduction}

Assisted reproductive techniques using frozen embryo transfer (FET) are becoming more common. Cryopreservation of embryos

Received: May 14, 2018 · Revised: Jun 19, 2018 · Accepted: Jul 4, 2018 Corresponding author: Alar Aints

Institute of Clinical Medicine, Department of Obstetrics and Gynecology, University of Tartu, Tartu 51014, Estonia

Tel:+372-53904250 E-mail: Alar.Aints@ut.ee

* This research was funded by the Estonian Ministry of Education and Research (grant IUT34-16), by Enterprise Estonia (grant no. EU48695), the EU-FP7 Marie Curie Industry-Academia Partnerships and Pathways (IAPP, grant SARM, EU324509), and Horizon 2020 innovation program (grant WIDENLIFE, 692065).

This is an Open Access article distributed under the terms of the Creative Commons Attribution Non-Commercial License (http://creativecommons.org/licenses/by-nc/4.0/) which permits unrestricted non-commercial use, distribution, and reproduction in any medium, provided the original work is properly cited. helps increase the cumulative pregnancy rate in in vitro fertilization (IVF) cycles [1]. However, embryo cryopreservation can cause zona pellucida (ZP) hardening, which leads to impaired embryo hatching and a lower implantation rate [2,3]. Scanning electron microscopy data have shown that the number and size of holes in the ZP of vitrified embryos were smaller than in fresh ZPs [4]. Other studies have shown that enzymatic digestion of the ZP with pronase in cryopreserved embryos required more time than in fresh embryos [5].

The implantation potential of a frozen/thawed embryo is lower than that of a fresh embryo. One of the reasons for this is that vitrification may impair hatching of the embryo. Theoretically, assisted hatching $(\mathrm{AH})$ may facilitate the hatching process and subsequently increase the implantation rate $[2,3,6]$. Many authors have suggested that perforating or thinning the ZP of frozen-thawed embryos by $\mathrm{AH}$ 
facilitates embryonic implantation by making it easier for the embryo to exit the surrounding ZP $[7,8]$.

$\mathrm{AH}$ has been used in humans since the 1990s [9]. There are four main techniques for $\mathrm{AH}$ : mechanical partial ZP dissection with glass pipettes, chemical ZP opening using acidic Tyrode solution, enzymatic ZP thinning using pronase, and diode laser-assisted hatching (LAH) [7]. The first three methods require manual or motorized micromanipulators and skilled embryologists, and carry a risk of direct mechanical injury or toxicity. Research has shown LAH to be more effective, simpler, and faster than mechanical and chemical methods [6]. Non-contact laser systems using infrared wavelengths $(1.4 \mu \mathrm{m})$ are commonly used in LAH today. This enables easy automated operation and avoids adverse effects, as the thermal effects on the embryo are negligible. LAH is performed by two methods: drilling or thinning. In drilling LAH (D-LAH), a laser beam is used to create a hole $20-40 \mu \mathrm{m}$ in diameter in the ZP, putting the embryo in direct contact with the culture medium. Embryos can easily hatch after they develop [10]. However, this method has disadvantages, including the risk of losing blastomeres through the opening and the direct contact of an embryo with high concentrations of nutrients and antibiotics in the environment [11]. In thinning LAH (T-LAH), a quarter to half the ZP is thinned from the outside, causing blastomeres to be less affected by heating. The size of the ZP thinning area in LAH affects the outcomes of vitrified-warmed embryo transfer (ET) at the cleavage stage [12]. Drilling a large enough hole in the ZP makes a clear path for the embryo to hatch. Thinning removes only $50 \%$ of the ZP thickness, and the embryo must break down the rest. Complete hatching through a thinned ZP occurs more slowly than through a drilled ZP, and sometimes the embryo can be trapped when trying to hatch via two routes simultaneously [13]. Incomplete hatching of an embryo significantly affects the implantation process and subsequent development of the fetus [14]. Given the advantages and disadvantages of the D-LAH and T-LAH methods for $\mathrm{AH}$, we wished to study whether either of these techniques was advantageous for FET. No studies have yet compared the efficacy of these two methods of LAH on the results of FET.

\section{Methods}

\section{Research plan}

A randomized comparative study using thawed embryos on day 2 was planned and carried out from December 2014 to December 2016 at the Center for Reproductive Endocrinology and Infertility, Hue University of Medicine and Pharmacy, Hue, Vietnam. A total of 171 frozen-thawed ET cycles were analyzed and randomly divided into two groups by an odd-even rule, resulting in 86 cycles for T-LAH and 85 cycles for D-LAH.

\section{Patients}

All patients (age, 22-47 years; median, 34 years) agreed to the conditions of the study and to take part in it. This study was approved by the Ethics Committee of Hue University of Medicine and Pharmacy. All patients met the following criteria: (1) a maximum of three previous failed IVF-ET procedures, and (2) undergoing FET. The patients had been infertile for an average of 4.97 years (standard deviation [SD], 2.75; range, 1-16 years). The indications for IVF-ET were male factor infertility (41\%), tubal factor infertility (29\%), endometriosis (11\%), and unexplained infertility (19\%). The patients were randomized on the day of embryo thawing and transfer.

\section{Ovarian stimulation, oocyte retrieval, and intracytoplasmic sperm injection}

The stimulation protocols, oocyte recovery, and ET techniques were used as previously described $[3,15]$. Briefly, all patients were treated with controlled ovarian stimulation by a gonadotropin-releasing hormone antagonist protocol using a starting dose of $225 \mathrm{IU}$ of recombinant follicle-stimulating hormone (rFSH) (Gonal F; Merck Serono, Darmstadt, Germany). Cetrotide (0.1 mg, Merck Serono) was administered when at least one follicle reached $14 \mathrm{~mm}$ or on day 6 of $\mathrm{rFSH}$ stimulation. Oocyte retrieval was performed by ultrasound-guided follicle aspiration, 35-36 hours after human chorionic gonadotropin (hCG) administration. Oocytes were fertilized using intracytoplasmic sperm injection (ICSI). Embryos were cultured in G-TL Vitrolife medium (Vitrolife, Gothenburg, Sweden). Fertilization was verified at 16 18 hours after sperm injection. Embryo quality was evaluated at 40 44 hours after injection, and classified as G1 ( $\geq 4$ cells and $<10 \%$ fragmentation), G2 ( $\geq 4$ cells or $10 \%-20 \%$ fragmentation), or G3 ( $<4$ cells and $>25 \%$ fragmentation) [16]. Only good-quality embryos on day 2 (G1 or G2) were selected for transfer or cryopreservation.

\section{Cryopreservation}

Vitrification was performed using a Kitazato kit (Kitazato, Shizuoka, Japan). The selected day 2 embryos were equilibrated for 5 minutes in equilibration solution. The equilibrated embryos were transferred into vitrification solution 1 for 40 seconds and then vitrification solution 2 before being loaded onto the prepared cryotop. The cryotop was immediately placed in liquid nitrogen $\left(-196^{\circ} \mathrm{C}\right)$. The interval from being in vitrification solution 1 to being placed in liquid nitrogen did not exceed 1 minute. Each cryotop contained a maximum of 2 to 4 good-quality $(\mathrm{G} 1, \mathrm{G} 2)$ day 2 embryos.

\section{Thawing procedure}

The cryotop was thawed according to the Kitazato instructions. Briefly, a 4-step thawing protocol was used to remove the cryoprotectant, and the thawed embryos were cultured in a $20-\mu \mathrm{L}$ droplet of 
G-TL medium (Vitrolife) covered in $3 \mathrm{~mL}$ of OVOIL (Vitrolife) equilibrated at $37^{\circ} \mathrm{C}, 5.5 \% \mathrm{CO}_{2}$, and $5 \% \mathrm{O}_{2}$ for 2 hours. Embryo quality was then re-evaluated and $\mathrm{AH}$ was performed. The embryos were considered to have survived if $\geq 50 \%$ of the blastomeres were intact or had at least three viable cells $[17,18]$. The embryo survival rate was $99 \%$.

\section{LAH of embryos}

Embryo thawing was performed on the morning of ET, and LAH was carried out just 2 hours before ET. ZP thickness was recorded as the average thickness of all embryos from the same patient. AH of human embryos was performed with a non-contact 630- to 650-nm pilot laser, an infrared diode laser (1,480 nm, $400 \mathrm{~mW}$, $\times 40$ objectives; Saturn 5 Active; BioMedical Instruments, Zoellnitz, Germany). The laser system was attached to an inverted microscope Axio Observer A1 (ZEISS, Oberkochen, Germany) and was controlled by RI Viewer imaging software with digital laser targeting (BioMedical Instruments).

In the T-LAH group, the laser was set to have a pulse length of 0.450 $\mathrm{ms}$ to $0.650 \mathrm{~ms}$, firing in a contiguous area along the periphery of the $\mathrm{ZP}$, covering one-fourth of its circumference. In the D-LAH group, the laser was set to have a pulse length of $0.450 \mathrm{~ms}$ to $0.650 \mathrm{~ms}$, and was used to make a hole $40 \mu \mathrm{m}$ in diameter in the ZP. After the laser procedure, the embryos were cultured for 2 hours before transfer.

\section{Embryo transfer}

Before transfer into the uterus, day 2 embryos were soaked in Embryo Glue (Vitrolife) medium for 15-30 minutes and then transferred through a Kitazato catheter. A pregnancy test measuring serum $\beta$-hCG levels was performed 15 days after the 2-day ET. If the pregnancy test was positive, the patient was followed with serial ultrasound to determine fetal viability. The first ultrasound scan was performed 2 weeks after a positive $\beta$-hCG pregnancy test ( 4 weeks after ET). Clinical pregnancy was defined as the presence of a gestational sac and fetal heartbeat on transvaginal ultrasound. Ongoing preg- nancy was defined as the presence of a gestational sac and fetal heartbeat on transvaginal ultrasound until 12 weeks. When pregnancy occurred, luteal support was continued until 8 weeks of gestation (Crinone gel 8\%, one tube in the morning; Utrogestan 400 mg, as vaginal support in the evening; Progynova $2 \mathrm{mg}$, two tablets per day; and prenatal vitamins).

\section{Statistics}

Data were processed using Excel (Microsoft, Redmond, WA, USA) and R ver. 3.4.0 (R Foundation for Statistical Computing, Vienna, Austria). Continuous variables were presented as mean $\pm S D$, and statistical significance was calculated using the independent sample $t$-test. Binary data were analyzed using the Pearson chi-square test. Differences were considered significant if the $p$-value was less than 0.05 .

\section{Results}

Data were collected from 171 IVF/ICSI cycles randomly divided into D-LAH (85 cycles with 223 embryos) and T-LAH (86 cycles with 243 embryos) groups. The groups were not significantly different regarding the age of the female partner, body mass index, or the number of embryos transferred. In the T-LAH group, 243 embryos were transferred and 40 (16.5\%) implanted. In the D-LAH group, 223 embryos were transferred and 32 (14.4\%) implanted. As shown in Table 1, no difference in the rate of $\beta$-hCG positivity was observed between the two groups. The results were similar in the T-LAH and D-LAH groups in terms of implantation and clinical pregnancy rate.

The median thickness of the ZP in our study group was $16.9 \mu \mathrm{m}$. We analyzed the differences between two subgroups defined based on the ZP thickness (greater than or less than $17 \mu \mathrm{m}$ ). We observed no differences in the $\beta$-hCG positivity rate, clinical pregnancy rate, ongoing pregnancy rate, or multiple pregnancy rate between the two subgroups defined based on ZP thickness (Table 2).

Table 1. Basal characteristics and main outcomes

\begin{tabular}{lcccc}
\hline Characteristics & D-LAH $(\mathrm{n}=85$ cycles $)$ & T-LAH $(\mathrm{n}=86$ cycles $)$ & Total $(\mathrm{n}=171$ cycles $)$ & $p$-value \\
\hline Age $(\mathrm{yr})$ & $33.8 \pm 5.4$ & $34.0 \pm 3.9$ & $33.9 \pm 4.7$ & $0.800^{\mathrm{b})}$ \\
BMI $\left(\mathrm{kg} / \mathrm{m}^{2}\right)$ & $20.5 \pm 2.6$ & $20.4 \pm 2.2$ & $20.5 \pm 2.4$ & $0.880^{\mathrm{b})}$ \\
Total embryo transferred/cycle (mean \pm SD) & $223 / 85(2.6 \pm 0.7)$ & $243 / 86(2.8 \pm 0.6)$ & $466 / 171(2.7 \pm 0.7)$ & 0.542 \\
$\beta$-hCG positive & $29.4(25 / 85)$ & $38.4(33 / 86)$ & $33.9(58 / 171)$ & 0.217 \\
Implantation rate & $14.4(32 / 223)$ & $16.5(40 / 243)$ & $15.5(72 / 466)$ & 0.529 \\
Clinical pregnancy rate & $25.9(22 / 85)$ & $36.0(31 / 86)$ & $31.0(53 / 171)$ & 0.152 \\
Early miscarriage rate & $2.4(2 / 85)$ & $5.8(5 / 86)$ & $4.1(7 / 171)$ & 0.255 \\
Ongoing pregnancy rate & $23.5(20 / 85)$ & $30.2(26 / 86)$ & $26.9(46 / 171)$ & 0.324 \\
Multiple pregnancy rate & $10.6(9 / 85)$ & $7.0(6 / 86)$ & $8.8(15 / 171)$ & 0.405 \\
\hline
\end{tabular}

Values are presented as mean \pm standard deviation (SD) or percentage (number of cycles or embryos) unless otherwise indicated. D-LAH, drilling laser-assisted hatching; T-LAH, thinning laser-assisted hatching; BMl, body mass index; hCG, human chorionic gonadotropin. ${ }^{\text {a) }}$ Chi-square test; ${ }^{\text {b) }}$ Two-sampled $t$-test. 
Table 2. Pregnancy outcomes related to zona pellucida thickness

\begin{tabular}{|c|c|c|c|c|c|c|}
\hline \multirow{2}{*}{ Pregnancy outcome } & \multicolumn{3}{|c|}{$<17 \mu \mathrm{m}$ ( $\mathrm{n}=86$ cycles $)$} & \multicolumn{3}{|c|}{$\geq 17 \mu \mathrm{m}$ ( $\mathrm{n}=85$ cycles $)$} \\
\hline & D-LAH & T-LAH & $p$-value ${ }^{a)}$ & D-LAH & T-LAH & $p$-value ${ }^{a}$ \\
\hline$\beta$-hCG positive & $33.3(16 / 48)$ & $47.3(18 / 38)$ & 0.189 & $24.3(9 / 37)$ & $31.2(15 / 48)$ & 0.484 \\
\hline Implantation rate & $16.1(20 / 124)$ & $16.0(17 / 106)$ & 0.985 & $12.1(12 / 99)$ & $16.8(23 / 137)$ & 0.321 \\
\hline Clinical pregnancy rate & $27.0(13 / 48)$ & $42.1(16 / 38)$ & 0.146 & $24.3(9 / 37)$ & $31.2(15 / 48)$ & 0.484 \\
\hline Early miscarriage rate & $2.0(1 / 48)$ & $13.2(5 / 38)$ & 0.046 & $2.7(1 / 37)$ & 0 & $0.435^{\mathrm{b})}$ \\
\hline Ongoing pregnancy rate & $25.0(12 / 48)$ & $28.9(11 / 38)$ & 0.683 & $21.0(8 / 37)$ & $31.2(15 / 48)$ & 0.325 \\
\hline Multiple pregnancy rate & $14.6(7 / 48)$ & $2.6(1 / 38)$ & 0.059 & $5.4(2 / 37)$ & $10.4(5 / 48)$ & 0.407 \\
\hline
\end{tabular}

Values are presented as percentage (number of cycles or embryos).

D-LAH, drilling laser-assisted hatching; T-LAH, thinning laser-assisted hatching; $\mathrm{hCG}$, human chorionic gonadotropin.

${ }^{\text {a) }}$ Chi-square test; ${ }^{\text {b) }}$ Fisher exact test.

Table 3. Pregnancy outcomes related to age

\begin{tabular}{|c|c|c|c|c|c|c|}
\hline \multirow{2}{*}{ Pregnancy outcome } & \multicolumn{3}{|c|}{$\leq 34 \mathrm{yr}$ ( $\mathrm{n}=96$ cycles $)$} & \multicolumn{3}{|c|}{$\geq 35$ yr ( $n=75$ cycles) } \\
\hline & D-LAH & T-LAH & $p$-value $e^{a)}$ & D-LAH & T-LAH & $p$-value ${ }^{a}$ \\
\hline$\beta$-hCG positive & $23.4(11 / 47)$ & $40.8(20 / 49)$ & 0.069 & $36.8(14 / 38)$ & $35.1(13 / 37)$ & 0.878 \\
\hline Implantation rate & $12.2(15 / 123)$ & $15.6(22 / 141)$ & 0.427 & $17.0(17 / 100)$ & $17.7(18 / 102)$ & 0.904 \\
\hline Clinical pregnancy rate & $21.3(10 / 47)$ & $36.7(18 / 49)$ & 0.097 & $31.6(12 / 38)$ & $35.1(13 / 37)$ & 0.746 \\
\hline Early miscarriage rate & $2.1(1 / 47)$ & $4.1(2 / 49)$ & 0.584 & $2.6(1 / 38)$ & $8.1(3 / 37)$ & 0.294 \\
\hline Ongoing pregnancy rate & $29.2(9 / 47)$ & $32.7(16 / 49)$ & 0.134 & $28.9(11 / 38)$ & $27.0(10 / 37)$ & 0.854 \\
\hline Multiple pregnancy rate & $8.5(4 / 47)$ & $8.2(4 / 49)$ & 0.951 & $13.2(5 / 38)$ & $5.4(2 / 37)$ & 0.252 \\
\hline
\end{tabular}

Values are presented as percentage (number of cycles or embryos).

D-LAH, drilling laser-assisted hatching; T-LAH, thinning laser-assisted hatching; $\mathrm{hCG}$, human chorionic gonadotropin.

${ }^{a)}$ Chi-square test.

Table 3 shows the outcomes when subgroups of $\leq 34$-year-old (median, 31 years) and $\geq 35$-year-old (median, 38 years) were compared. The differences were not statistically significant. However, there was a trend for better results to be found for the clinical pregnancy rate in younger patients using thinning $(p=0.097)$.

\section{Discussion}

Previous studies have used a wide variety of $\mathrm{AH}$ techniques, and a meta-analysis of those studies produced few meaningful results due to the heterogeneity of the studies [19]. AH procedures vary in terms of time and mode of application, thereby resulting in differences in hatching dynamics. In our study, we compared two main methods of LAH: thinning and drilling. The effectiveness of these two methods has not been directly compared in frozen and thawed embryos, and varying results for their efficacy have been reported $[2,10,20]$. In our study, we focused on the effect of drilling and thinning on FET results.

Studies similar to our own have shown that the choice of the method of LAH (i.e., D-LAH vs. T-LAH) had no effect on the pregnancy rate $[14,21]$. In a previous study, the implantation rate was found to be similar $(p=0.44)$ in the T-LAH group $(n=19,34.5 \%)$ and the D-LAH group ( $n=111,29.4 \%)$ when fresh embryos were used [20]. In our study group, in which AH was performed on day 2 frozen and thawed embryos, the differences were not statistically significant between the thinning and drilling group in terms of the biochemical pregnancy rate $(38.4 \%$ vs. $29.4 \%)$ or the clinical pregnancy rate $(36.0 \%$ vs. $25.9 \%$ ) ( $p>0.2$ for both). However, a trend for thinning to be more effective, similar to the results of Padula et al. [20], was observed.

The thickness of the ZP can affect the embryo's hatching. The thicker the ZP, the harder it is for the embryo to hatch [11]. Several studies have shown that a ZP thickness of more than $15 \mu \mathrm{m}$ had a negative effect on embryo hatching and pregnancy outcomes, meaning that such cases need AH [22]. In our study group, the median ZP thickness was $16.9 \mu \mathrm{m}$, so we used $17 \mu \mathrm{m}$ as a cut-point for a subgroup analysis. The rate of success was similar in the T-LAH and D-LAH groups in both groups defined by ZP thickness (greater than or less than 17 $\mu \mathrm{m})$. The early miscarriage rate appeared to be higher for patients in the $<17 \mu \mathrm{m}$ group who underwent T-LAH (13.2\%, 5/38 for T-LAH vs. $2.1 \%, 1 / 48$ for D-LAH; $p=0.046)$. However, we believe that this is attributable to chance due to the low number of cases involved.

According to many studies, maternal age has a significant influence on the genetics and viability of the embryo. Unsuccessful hatching may affect the outcomes of ICSI-ET. AH methods are thought to be effective in improving the treatment outcomes for older patients 
[23]. In this study, we compared the efficacy of two AH methods in subgroups of patients $\leq 34$ and $\geq 35$ years of age. The differences were not statistically significant. However, a trend $(p=0.097)$ for a higher clinical pregnancy rate was demonstrated for T-LAH cycles in the younger age group, which represented more than half (56.1\%, 96/171) of the entire study cohort (Table 3). This difference was primarily due to the lower pregnancy rate in the D-LAH group.

The clinical pregnancy rate in our study is comparable to published results for the 25- to 35-year age group (35\%-36\%) [24]. The clinical pregnancy rate in the thinning group did not differ between age groups (36.7\% in those $\leq 34$ years of age vs. $35.1 \%$ in those $\geq 35$ years of age). It is possible that embryo hatching is one of the steps responsible for this difference and that $\mathrm{AH}$ eliminates it. Other factors acting later in pregnancy development reduce the success rate further for older patients, as can be seen from the lower ongoing pregnancy rates in the older group $(32.7 \%$ in those $\leq 34$ years of age vs. $27.0 \%$ in those $\geq 35$ years of age) although the difference was not significant. Other factors involved may be related to ethnicity or lifestyle.

In early studies of LAH, thinning was reported to be superior to drilling. Comparing those results to ours, it can be seen that this difference arose from very low rates of success in the drilling group (clinical pregnancy rate: D-LAH, 5.2\% vs. T-LAH, 22.1\%) [25]. Later reports confirmed that drilling is more technically demanding, requires greater operator skill, and can be damaging to the embryo [20]. In our study, the success rate was higher than the early results in both groups (clinical pregnancy rate: D-LAH, $25.9 \%$ vs. T-LAH, $36.0 \%$ ); the difference was not significant although T-LAH showed slightly better outcomes.

In conclusion, the present study demonstrated that there were no significant differences in the implantation and pregnancy rates when transferring thawed embryos after T-LAH or D-LAH procedures. ZP thickness and the women's age had no effect on the implantation and clinical pregnancy rates in women who underwent ICSI-FET and $\mathrm{AH}$ treatment by these two methods. Although there were no statistically significant differences between the two groups, the T-LAH group in which one-fourth of the ZP was thinned showed a tendency for better ET results than the D-LAH group. Thinning is also considered to be safer for the embryo and easier to perform [20]. However, the small sample size may have affected the results of this study, so studies of larger groups or pooling similar studies for meta-analysis may provide data that would reach statistical significance.

\section{Conflict of interest}

No potential conflict of interest relevant to this article was reported.

\section{References}

1. Veleva Z, Orava M, Nuojua-Huttunen S, Tapanainen JS, Martikainen $\mathrm{H}$. Factors affecting the outcome of frozen-thawed embryo transfer. Hum Reprod 2013;28:2425-31.

2. Elhussieny A, Mandouh M E, Hanafi S, Mansour GM, El-kotb A. Effect of laser assisted hatching on outcome of assisted reproductive technology. Open J Obstet Gynecol 2013;3:18-23.

3. Kanyo K, Zeke J, Kriston R, Szucs Z, Cseh S, Somoskoi B, et al. The impact of laser-assisted hatching on the outcome of frozen human embryo transfer cycles. Zygote 2016;24:742-7.

4. Gabrielsen A, Agerholm I, Toft B, Hald F, Petersen K, Aagaard J, et al. Assisted hatching improves implantation rates on cryopreserved-thawed embryos: a randomized prospective study. Hum Reprod 2004;19:2258-62.

5. Valojerdi MR, Eftekhari-Yazdi P, Karimian L, Hassani F, Movaghar B. Effect of laser zona thinning on vitrified-warmed embryo transfer at the cleavage stage: a prospective, randomized study. Reprod Biomed Online 2010;20:234-42.

6. Wang EH, Wang AC, Wang BS, Li B. Outcomes of vitrified-warmed cleavage-stage embryo hatching after in vitro laser-assisted zona pellucida thinning in patients. Biomed Rep 2016;5:376-82.

7. Balaban B, Urman B, Alatas C, Mercan R, Mumcu A, Isiklar A. A comparison of four different techniques of assisted hatching. Hum Reprod 2002;17:1239-43.

8. Ng EH, Naveed F, Lau EY, Yeung WS, Chan CC, Tang OS, et al. A randomized double-blind controlled study of the efficacy of laser-assisted hatching on implantation and pregnancy rates of frozen-thawed embryo transfer at the cleavage stage. Hum Reprod 2005;20:979-85.

9. Cohen J, Feldberg D. Effects of the size and number of zona pellucida openings on hatching and trophoblast outgrowth in the mouse embryo. Mol Reprod Dev 1991;30:70-8.

10. Link B, Wong B, Mayer J, Sullivan M, Fleetham J, Greene C. Laserassisted hatching (LAH) of cryopreserved embryos: the signifcance of hole size. Fertil Steril 2012;98:S79-80.

11. Hiraoka K, Fuchiwaki M, Hiraoka K, Horiuchi T, Murakami T, Kinutani $\mathrm{M}$, et al. Effect of the size of zona pellucida opening by laser assisted hatching on clinical outcome of frozen cleaved embryos that were cultured to blastocyst after thawing in women with multiple implantation failures of embryo transfer: a retrospective study. J Assist Reprod Genet 2008;25:129-35.

12. Blake DA, Forsberg AS, Johansson BR, Wikland M. Laser zona pellucida thinning: an alternative approach to assisted hatching. Hum Reprod 2001;16:1959-64.

13. Hiraoka K, Hiraoka K, Horiuchi T, Kusuda T, Okano S, Kinutani M, et al. Impact of the size of zona pellucida thinning area on vitri- 
fied-warmed cleavage-stage embryo transfers: a prospective, randomized study. J Assist Reprod Genet 2009;26:515-21.

14. Schimmel T, Cohen J, Saunders H, Alikani M. Laser-assisted zona pellucida thinning does not facilitate hatching and may disrupt the in vitro hatching process: a morphokinetic study in the mouse. Hum Reprod 2014;29:2670-9.

15. Zeke J, Konc J, Kanyo K, Kriston R, Cseh S. Birth and clinical pregnancy from fresh and frozen oocytes fertilized with cryopreserved testicular spermatozoa. Syst Biol Reprod Med 2012;58:165-7.

16. Ziebe S, Lundin K, Loft A, Bergh C, Nyboe Andersen A, Selleskog $\mathrm{U}$, et al. FISH analysis for chromosomes 13, 16, 18, 21, 22, X and $Y$ in all blastomeres of IVF pre-embryos from 144 randomly selected donated human oocytes and impact on pre-embryo morphology. Hum Reprod 2003;18:2575-81.

17. Ubaldi F, Rienzi L, Baroni E, Ferrero S, lacobelli M, Minasi MG, et al. Cumulative pregnancy rates after transfer of fresh and thawed embryos. Eur J Obstet Gynecol Reprod Biol 2004;115 Suppl 1:S106-9.

18. Rienzi L, Ubaldi F, Martinez F, Minasi MG, lacobelli M, Ferrero S, et al. Clinical application of laser-assisted ICSI: a pilot study. Eur J Obstet Gynecol Reprod Biol 2004;115 Suppl 1:S77-9.

19. Alteri A, Vigano P, Maizar AA, Jovine L, Giacomini E, Rubino P. Re- visiting embryo assisted hatching approaches: a systematic review of the current protocols. J Assist Reprod Genet 2018;35:367-91.

20. Padula F, Capriglione S, laconianni P, Gatti S, Lippa A, Minutolo $\mathrm{SE}$, et al. Laser-assisted hatching of human embryos: may two alternative approaches (thinning versus drilling) impact on implant rate? Lasers Med Sci 2017;32:1663-6.

21. Cohen J, Alikani M. Evidence-based medicine and its application in clinical preimplantation embryology. Reprod Biomed Online 2013;27:547-61.

22. Hammadeh ME, Fischer-Hammadeh C, Ali KR. Assisted hatching in assisted reproduction: a state of the art. J Assist Reprod Genet 2011;28:119-28.

23. Ghannadi A, Kazerooni M, Jamalzadeh F, Amiri S, Rostami P, Absalan F. The effects of laser assisted hatching on pregnancy rates. Iran J Reprod Med 2011;9:95-8.

24. Humm KC, Dodge LE, Wu LH, Penzias AS, Malizia BA, Sakkas D, et al. In vitro fertilization in women under 35: counseling should differ by age. J Assist Reprod Genet 2015;32:1449-57.

25. Mantoudis E, Podsiadly BT, Gorgy A, Venkat G, Craft IL. A comparison between quarter, partial and total laser assisted hatching in selected infertility patients. Hum Reprod 2001;16:2182-6. 